\title{
Probabilistic Assessment of Net Transfer Capacity Considering Forecast Uncertainties
}

\author{
F. Adinolfi, S. Massucco \\ M. Saviozzi, F. Silvestro \\ University of Genova - DITEN \\ Intelligent Energy Systems Laboratory \\ Genoa, Italy \\ stefano.massucco@unige.it
}

\author{
E. Ciapessoni, D. Cirio \\ A. Pitto \\ RSE - Ricerca Sistema Energetico \\ Milan, Italy \\ andrea.pitto@rse-web.it
}

\begin{abstract}
In transmission system planning, researchers propose methods to assess the effect of uncertainties of power system operating condition due to forecasting errors of intermittent generation and loads. In particular probabilistic power flow methods are used to calculate the probability distributions of the voltages and the branch currents, starting from the distributions of power injections/absorptions. These uncertainties play a key role in the operational planning of power systems, as certain configurations of load and intermittent generation can cause security problems. This paper aims to propose a probabilistic methodology to assess Net Transfer Capacity (NTC) among network areas, which quantifies forecast error uncertainties by applying the Point Estimate Method (PEM) combined with Third Order Polynomial Normal (TPN) Transformation. This approach is compared with a conventional NTC assessment technique and has been tested on an IEEE test system.
\end{abstract}

Index Terms - net transfer capacity, probability, point estimate method, third order polynomial normal transformation

\section{INTRODUCTION}

The increasing uncertainties in power systems originated by liberalized market and the penetration of Renewable Energy Sources (RES) raise more and more attention to security issues [1]-[4]. RES forecast uncertainties are affected by several factors, e.g. prediction techniques, RES aggregation level and forecast horizon: correlations in RES and load forecast errors may heighten the deviation of the operating condition with respect to the forecast state, possibly exacerbating security issues [5], [6]. Evaluation of Net Transfer Capacity (NTC) between contiguous areas in a network is also affected by this issue. Guidelines for the NTC computation are provided by operational standards [7]. Current approaches rely on the adoption of deterministic security margins for NTC evaluations. Probabilistic approaches would be more accurate, possibly leading to increased market efficiency (in case of less conservative results) or security (in case of security issues associated to the specific uncertainty patterns). Up to now, research has focused on the calculation of ATC (Available Transfer Capacity, equal to $\mathrm{NTC}-\mathrm{AAC}$, where AAC = Already Allocated Capacity, i.e. the total of the allocated transmission rights) proposing two types of methods: deterministic methods and probabilistic methods [8]. Probabilistic methods are usually based on the combination of Monte Carlo simulation with a sensitivity analysis [9]-[12]. Moreover, the establishment of an integrated European electricity market underlines the importance to consider the physical characteristics and the constraints of the network by switching from an ATC based market (where individual TSOs compute the maximum bilateral cross-border flows between their area and each of neighboring areas with assumptions on potential parallel flows) to a flow-based market (accounting for physical limits of the cross-border lines, thus also for the loop flows), which will assure larger market opportunities [13].

In this context, the present work provides a novel probabilistic method to calculate the Net Transfer Capacity (NTC) among areas accounting for RES and load forecast uncertainties with their correlations, and for the constraints of the grid (modeled via a full AC power flow model). The proposed method, implemented in the Integrated Security assessment Platform (ISAP) [13], is based on coupling the Point Estimate Method (PEM) [15] scheme with Third order Polynomial Normal (TPN) Transformation [16], which represents an original contribution in the context of NTC studies. The rest of the paper is organized as follows: Section II introduces some definitions and the conventional NTC calculation procedure. Section III presents the probabilistic NTC methodology accounting for RES and load forecast error uncertainties. Section IV describes the test system and the simulation scenarios, and it reports and discusses the results. Section V concludes, while Appendix describes PEM and TPN Transformation in detail.

\section{Net And Total TRAnsfer CAPACITY: DeFINITIONS AND CONVENTIONAL ASSESSMENT TECHNIQUE}

After briefly recalling some definitions, the section describes the basic procedure for Total Transfer Capacity (TTC) evaluation and the conventional method to calculate the NTC. 


\section{A. Net and Total Transfer Capacity Definitions}

The definition of transmission capacities is given by European network of Transmission System Operators for Electricity (ENTSO-E) in [17] and it is briefly recalled here:

- Total Transfer Capacity (TTC) is the maximum exchange programme between two contiguous areas compatible with operational security standards, applicable to each system if future network conditions, generation and load patterns were perfectly known in advance.

- Transmission Reliability Margin (TRM) is a security margin that copes with uncertainties on the computed TTC values.

- $\quad$ Net Transfer Capacity (NTC) is the maximum exchange programme between two contiguous areas compatible with security standards applicable in both areas and taking into account the technical uncertainties on future network conditions.

NTC is evaluated according to the following formula:

NTC $=$ TTC - TRM

TTC is determined by physical constraints that may limit the system operation according to security rules [7]: (a) current limits; (b) voltage limits; (c) stability limits (due to frequency, voltage and angle instability). These limits must be respected also in post-contingency conditions, according to the operating security criteria (typically N-1 criterion). TTC evaluation is mostly influenced by: (1) the capability of the single connections of the considered network corridor; (2) The geographic location of the generators and loads with respect to the above mentioned corridor.

\section{B. Basic Algorithm for TTC Evaluation and Conventional NTC Assessment}

The basic procedure exploited in this work to evaluate the TTC is taken from [7] and it is based on the definition of a reference scenario. This scenario can be determined by optimal power flow technique or can be defined by the Transmission System Operator in order to study a real case.

Let $\mathrm{A}$ and $\mathrm{B}$ be the two areas under investigation for TTC evaluation. Starting from a reference scenario, the generated power is shifted from one area to the other in order to cause additional cross-border flows. This is done by increasing the generation in A step by step and decreasing the generation in B by the same steps, thus obtained a new grid operating state. The modification of the generator set-points doesn't involve renewable sources or CHP (combined Heat and Power) plants, but only the dispatchable conventional hydro and thermal units. The consumer loads in both areas remain unchanged. The generation stress is performed until one of the following conditions occurs in the new operating state:

- the generation variation in one area cannot be compensated by the generation in the second area due to the attainment of generators minimum or maximum active power limits

- static security violations in $\mathrm{N}$ condition

- static security violations during N-1 analysis performed by explicitly considering all individual branch outages.
A dichotomic algorithm is used to determine the maximum stress which does not cause any security violations, due to its robustness and computational efficiency. Security violations both in $\mathrm{N}$ and $\mathrm{N}-1$ states are verified by running the power flow on a full AC model of the complete grid: modeling also the areas not involved in the generation stress allows to detect potential bottlenecks all over the grid, without any "ex-ante" assumptions on parallel flows (as in the capacity allocations for current ATC based markets).

Up to now, dynamic security assessment is not included in the TTC calculation procedure. Some dynamic simulations may be run in the final operating point, to check the fulfillment of dynamic security requirements. The conventional procedure for the NTC evaluation, implemented in this work, is based on equation (1): after the deterministic TTC evaluation a TRM value, equal to the $5 \%$ of TTC, is applied.

\section{The Proposed NTC Calculation Method}

The conventional NTC evaluation proposed in the previous section seems pragmatic; however, the reliability margin does not explicitly depend on the k-hour ahead RES and load forecast errors referring to the scenarios studied in operational planning sessions. The proposed probabilistic evaluation intends to better evaluate the NTC, by quantifying the effect of RES and load forecasting errors. The proposed methodology is based on two steps:

1. Build the Cumulative Distribution Function (CDF) of TTC considering the RES/loads forecasting errors;

2. Compute the probabilistic NTC, here defined as the value of TTC which has a probability of being overcome equal to $\alpha$ : $\operatorname{NTC}_{\alpha}=\operatorname{CDF}^{-1}(1-\alpha)$. This definition of NTC appears more consistent with the operating reality, provided that a good forecast error model is available. This may be increasingly the case with TSOs (Transmission System Operators), as RES forecast techniques get better and better.

\section{A. Probabilistic modeling of the forecast errors}

Statistical dependences among the forecast errors may increase problem complexity. To this aim, two aspects are analysed in detail:

A. The marginal distributions of the forecast errors of RES and loads

B. Possible dependences among the forecast errors

As for point $\mathrm{A}$, the marginal distributions for the $\mathrm{k}$-hour ahead forecast errors of RES (solar and wind plants) can be derived from climatological models or from more advanced models like ensemble forecasts [4]. The approach presented in the simulations implements models drawn from different studies [3]. In particular, the non-symmetry of the forecast errors, derived from statistical analyses of historical data, suggests the use of non-symmetric distributions (like beta distributions) for wind and solar generation forecast errors. It is worth remarking that systematic errors in forecasts may determine a non-null mean value for the forecast errors, especially with regard to RES generation. 
The standard deviation of both wind and solar power generation forecast errors depends on [18][19]:

- Level of aggregation of RES: the larger is the number of wind/solar farms aggregated into the same "equivalent" generation, the lower is the standard deviation

- Geographic extension of the RES aggregation: given the same number of aggregated RES sources, the larger the area where they are distributed the higher the compensation effect among RES, thus the smaller is the standard deviation expressed in $\%$ of the total rating

- The forecast time horizon: typically the larger the time horizon the larger the variance in forecast errors.

To account for the first two aspects the model starts from a simplified representation of the "geographical extension" of the RES plants aggregated into each equivalent injection, depicted by an equivalent radius $r$, the number $N$ and the individual standard deviation $\sigma$, in $\mathrm{MW}$, for each involved machine (distinguishing one $\sigma_{S P}$ value for all solar panels and one $\sigma_{W T}$ value for all wind turbines). As an example, the standard deviation $\sigma_{W}^{T O T}$ of the aggregated wind power injection is evaluated as in (2).

$\sigma_{W}^{T O T}=\sqrt{v_{W T} \cdot \Sigma \cdot v_{W T}^{T}}$

where $v_{W T}$ is a vector $(1 \times N) \quad\left[\sigma_{W T} \ldots \sigma_{W T}\right]$ of the standard deviations of the individual units, $\Sigma$ is the correlation matrix among individual injections and it is calculated by two steps:

1. The $N^{2} / 2-N$ distances $d_{j}$ among the units (i.e. the offdiagonal terms of the upper triangular matrix related to matrix $\Sigma$ ) are randomly extracted with a uniform distribution within the circle with radius $r$.

2. For each distance $d_{j}$ the corresponding linear correlation coefficient $\rho_{j}$ is calculated taking into account also the time horizon $T$ under study using the relationship in (3) derived for wind farms from [18].

$\rho_{j}=e^{-d_{j} / H}$

where $H=a \cdot T+b$, with $a, b$ parameters tuned on the basis of experimental data in [18] using the method of least squares.

The standard deviation of the solar forecast error is expressed as a \% of the maximum producible power $P_{\max G \text {, }}$ which depends on the period of the year (seasonal effect) and of the day (daylight/night), and is different from rated power of the plant. The calculation of $P_{\max G}$ is addressed in [19].

Standard deviation of the forecast error also depends on the so called clearness index $C I$ (the ratio between actual solar generation $\mathrm{P}_{\mathrm{G}}$ and maximum producible solar power); in fact, in a sunny day or in a completely cloudy day, forecasting solar generation is not affected by errors. Given the standard deviation $\sigma_{\max C I}$ of the error for the maximum $C I$ condition, Fig. 1 provides the derating factor $D F$-see (4)- to be applied to $\sigma_{\max C I}$ as a function of $C I$, derived from [20].

$$
\begin{aligned}
& D F=9.74 \cdot C I^{4}-15.06 \cdot C I^{3}+1.75 \cdot C I^{2}+ \\
& +3.61 \cdot C I-0.015
\end{aligned}
$$

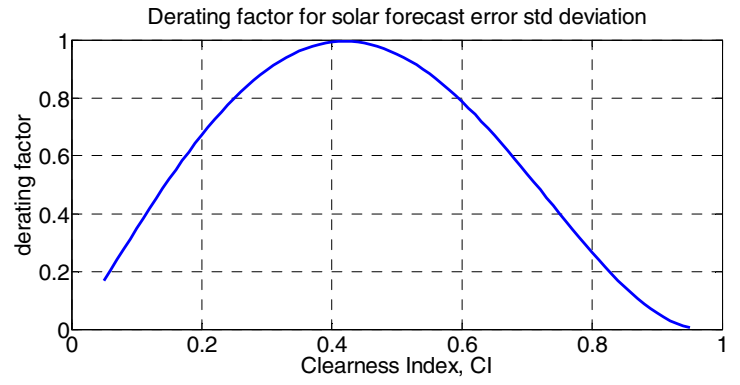

Fig. 1. Derating factor for the standard deviation $\sigma_{\operatorname{maxCI}}$ of the solar forecast error at maximum $\mathrm{CI}$ as a function of $\mathrm{CI}$

Load forecast errors are usually represented as normal distributions with usually low standard deviations (typically $1-4 \%$ of the actual power).

As for the modelling of dependences among stochastic inputs, different techniques, like the Nataf transformation and the TPN transformation [15], have been proposed in literature to simulate the behaviour of dependent stochastic variables without using time consuming Monte Carlo techniques. The present work adopts the TPN transformation to model input dependencies, as the input distributions may significantly differ from the normal distribution. More details about this technique can be found in Appendix.

\section{B. The algorithm for NTC calculation}

The following algorithm describes the application of the PEM technique combined with the TPN Transformation to assess the TTC distribution. Different PEM schemes have been proposed in literature to address Probabilistic Power Flow. The present approach assumes a $2 m+1$ PEM scheme (a suitable tradeoff between accuracy and computational burden) where $m$ is the number of stochastic variables. The algorithm consists in the following steps:

a) According to PEM scheme generate $2 * m+1$ input vectors $V$ and the corresponding weights $W$ for $m$ transformed independent normal variables $Y$

b) Calculate the $L$-moments $\ell_{i}(\mathrm{i}=1, \ldots, 4)$ and coefficients $a_{i}$ related to original variables $X$ as detailed in Appendix

c) Calculate correlation matrix $\rho_{Z}$ of the transformed dependent normal variables $Z$

d) Calculate matrix $G_{Z}$ as the result of a Cholesky decomposition of the correlation matrix of the transformed variables $Z$

e) Using matrix $G_{Z}$, translate the $2 m+1$ points at step $a$ in terms of transformed dependent variables $Z$

f) Use third order polynomial expression $x_{i}=a_{0, i}+a_{1, i} \cdot Z_{i}+a_{2, i} \cdot Z_{i}^{2}+a_{3, i} \cdot Z_{i}^{3}$ for all variables $i=1, \ldots, m$ and evaluate the $2 m+1$ points in terms of original variables $X$

g) For each $i$-th input vector of the method, apply the TTC conventional calculation and find the TTC value for the $i$ th PEM state $T T C^{(i)}$

h) Get the moments of the distributions of the TTC using weights $W$ 
i) Calculate central moments and cumulants [22] of the outputs from the raw moments

j) Reconstruct the probability distributions of the TTC using Gram-Charlier series expansion [22] which translates the TTC density into a linear combination of a normal density and its derivatives, with coefficients computed on the basic of cumulant

k) Compute NTC as: $\mathrm{NTC}_{\alpha}=\mathrm{CDF}^{-1}(1-\alpha)$

Parameter $\alpha$ can be evaluated by each TSO based on its yearly targets for security requirements imposed by the regulatory framework, taking into account on one side the congestion costs due to market separation, and on the other side the costs and the amount of reserve to keep congestion hours below a specific limit.

The proposed approach has been validated against a benchmark method (Monte-Carlo) in [21]: results show a good matching between the two methods, and significant computational time savings by using the proposed method instead of Monte-Carlo.

\section{Test System And Simulation Results}

\section{A. RTS-96 description \& stress scenarios}

The proposed algorithm for the NTC evaluation has been tested on IEEE RTS-96 network [23]. Its one-line diagram is reported in Fig. 2. This network is divided into 3 macroareas: in turn, each macroarea has two areas at different voltage levels, respectively $138 \mathrm{kV}$ and $230 \mathrm{kV}$. All $230 \mathrm{kV}$ areas are labeled with even numbers, while all $138 \mathrm{kV}$ areas are labeled with odd numbers. For simulation purposes, six conventional units have been replaced with three wind farm injections and three solar plant injections (shown in Fig. 2) with equivalent ratings and active power set-points so that the original loadflow is not altered. TABLE I reports the data for probabilistic characterization of RES generation.

TABLE I.

DATA FOR STOCHASTIC CHARACTERIZATION OF RES GENERATION

\begin{tabular}{|l|c|c|c|c|c|}
\hline & $\begin{array}{l}\text { Total } \\
\text { rating } \\
{[\mathrm{MW}]}\end{array}$ & $\begin{array}{l}\text { Number } \\
\text { of devices }\end{array}$ & $\begin{array}{l}\text { Equivalent } \\
\text { area radius, } \\
r \\
{[\mathrm{~km}]}\end{array}$ & $\begin{array}{l}\text { Std dev of } \\
\text { forecast } \\
\text { error*,\% of } \\
\text { device rated } \\
\text { power }\end{array}$ & $\begin{array}{l}\text { Mean value } \\
\text { of forecast } \\
\text { error* \% of } \\
\text { device rated } \\
\text { power }\end{array}$ \\
\hline W1 & 355 & 78 & 15 & 15 & 0 \\
\hline W2 & 355 & 78 & 15 & 15 & 0 \\
\hline W3 & 355 & 78 & 15 & 15 & 0 \\
\hline PV1 & 276 & 2000 & 15 & 25 & 0 \\
\hline PV2 & 230 & 1000 & 15 & 25 & 0 \\
\hline PV3 & 250 & 1520 & 15 & 25 & 0 \\
\hline
\end{tabular}

*referred to a single component (WT or SP) and to a 24-hour ahead horizon

The rating and the actual injections of RES units have been increased by $200 \mathrm{MW}$ with respect to original unit ratings in [23] to increase the effect of uncertainties: this has implied a corresponding increase in loads at the same connection points of RES to maintain the same powerflows. Zero means are assumed for all RES forecast error models. The loads and the generators set-points have been reduced by a factor equal to $2 / 3$ with respect to the RTS-96 configuration in [23]. The sum of the rated powers of RES equivalent injections covers about $15 \%$ of the total active load.

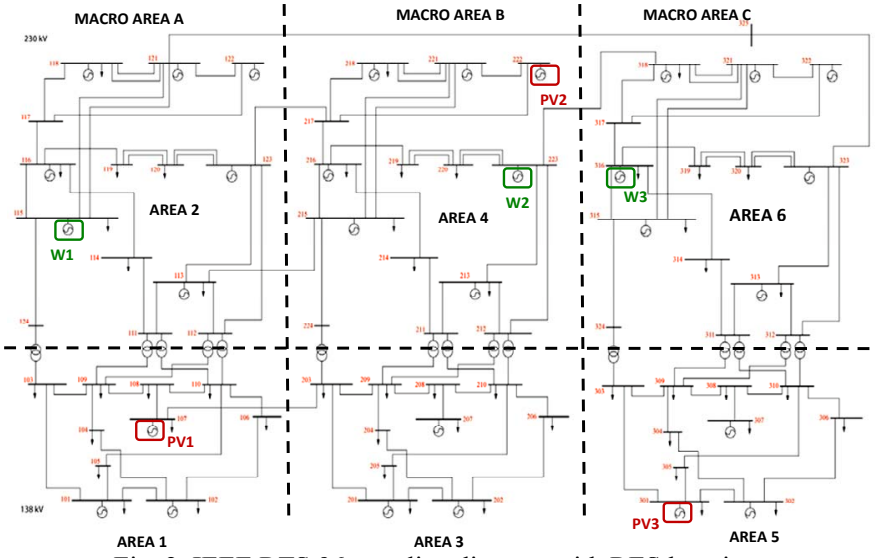

Fig. 2. IEEE RTS 96: one line diagram with RES location

Two Stress Directions (SD) are considered in the present paper and are listed in TABLE II. Columns "Area UP" and "Area Down" of TABLE II identify respectively the generation increase area and the generation decrease area.

TABLE II.

STRESS DIRECTIONS AND CONVENTIONAL ANALYSIS RESULTS

\begin{tabular}{l|lllll}
\hline SD & Area & Area & TTC & NTC & Stop \\
& UP & DOWN & {$[M W]$} & {$[M W]$} & criterion \\
\hline A & 4 & 6 & 367 & 348 & N-1 analysis \\
B & $3-4$ & $1-2$ & 762 & 724 & N-1 analysis \\
\hline
\end{tabular}

Conventional TTC calculation assumes a tolerance between the last secure and the first insecure generation increment in the dichotomy algorithm is set to $2 \mathrm{MW}$. The TRM value is set to $5 \%$ of the corresponding TTC value.

In particular, the TTC for scenario A is limited by the overload of branch between nodes 223 and 318 due to the outage of branch 121-325; the TTC calculated for scenario B is constrained by the overload of branch connecting nodes 113 and 215 due to the outage of branch 123-217.

Different simulation scenarios for the two stress directions "SD" are simulated to test the ability of the proposed methodology to account for different aspects, like the distance between RES plants, the time horizon, but also the period of the year and of the day which affects the standard deviation of the solar generation forecast errors. TABLE III summarizes the scenarios.

TABLE III.

SIMULATION SCENARIOS

\begin{tabular}{|l|l|l|l|l|l|l|}
\hline ID & $\begin{array}{l}\text { Distance } \\
\text { RES, km }\end{array}$ & $\begin{array}{l}\text { Time } \\
\text { horizon } \\
{[\mathrm{h}]}\end{array}$ & $\begin{array}{l}\text { WFs } \\
\text { (sign } \\
\text { corr.) }\end{array}$ & $\begin{array}{l}\text { PVs (sign } \\
\text { corr.) }\end{array}$ & Month/hour & SD \\
\hline 1 & 20 & 24 & $2-3(+)$ & $2-3(+)$ & $\mathrm{Aug} / 1 \mathrm{pm}$ & $\mathrm{A}$ \\
\hline 2 & 20 & 24 & $2-3(-)$ & $2-3(-)$ & $\mathrm{Aug} / 1 \mathrm{pm}$ & $\mathrm{A}$ \\
\hline 3 & 200 & 24 & $2-3(+)$ & $2-3(+)$ & $\mathrm{Aug} / 1 \mathrm{pm}$ & $\mathrm{A}$ \\
\hline 4 & 20 & 12 & $2-3(+)$ & $2-3(+)$ & $\mathrm{Aug} / 1 \mathrm{pm}$ & $\mathrm{A}$ \\
\hline 5 & 20 & 24 & $2-1(+)$ & $2-1(+)$ & $\mathrm{Aug} / 1 \mathrm{pm}$ & $\mathrm{B}$ \\
\hline 6 & 20 & 24 & $2-1(-)$ & $2-1(-)$ & $\mathrm{Aug} / 1 \mathrm{pm}$ & $\mathrm{B}$ \\
\hline 7 & 200 & 24 & $2-1(+)$ & $2-1(+)$ & $\mathrm{Aug} / 1 \mathrm{pm}$ & $\mathrm{B}$ \\
\hline 8 & 20 & 12 & $2-1(+)$ & $2-1(+)$ & $\mathrm{Aug} / 1 \mathrm{pm}$ & $\mathrm{B}$ \\
\hline 9 & 20 & 24 & $2-3(+)$ & $2-3(+)$ & $\mathrm{Mar} / 1 \mathrm{pm}$ & $\mathrm{A}$ \\
\hline 10 & 20 & 24 & $2-1(+)$ & $2-1(+)$ & $\mathrm{Mar} / 1 \mathrm{pm}$ & $\mathrm{B}$ \\
\hline
\end{tabular}


The header "Distance RES" refers to the distance in $\mathrm{km}$ among the WF's and PV plants mentioned in the relevant columns. All other RES plants are assumed to be far from one another (negligible correlation), this has been done to separate the effect of each of the abovementioned influencing factors.

\section{B. Effect of RES correlation and time horizon}

Correlation among equivalent RES injections affects the probability distributions of branch powerflows, thus affecting also the TTC CDF. Fig. 3 compares the TTC CDF's related to scenarios 1 and 2. By comparing scenario 1 and 2 for stress A, and 5 and 6 for stress B, it can be noticed that passing from a high positive (e.g. +0.75 between $\mathrm{W} 2$ and W3 injections in A1) to a high negative correlation $(-0.75$ between $\mathrm{W} 2$ and $\mathrm{W} 3$ in A1) among the RES plants on different sides of the analysed interconnection determines a decrease of the standard deviation of the TTC curve. The reason is that a positive correlation means a higher probability of concordant variations of RES injections in the UP and DOWN areas of the stress direction, which in turn causes a larger displacement of conventional generation production with respect to the original base case, thus a larger dispersion of the powerflow along the interconnections under study. The estimated NTC (as 10\% quantile of CDF) passes from 352 (positive corr.) to $356 \mathrm{MW}$ (negative corr.).

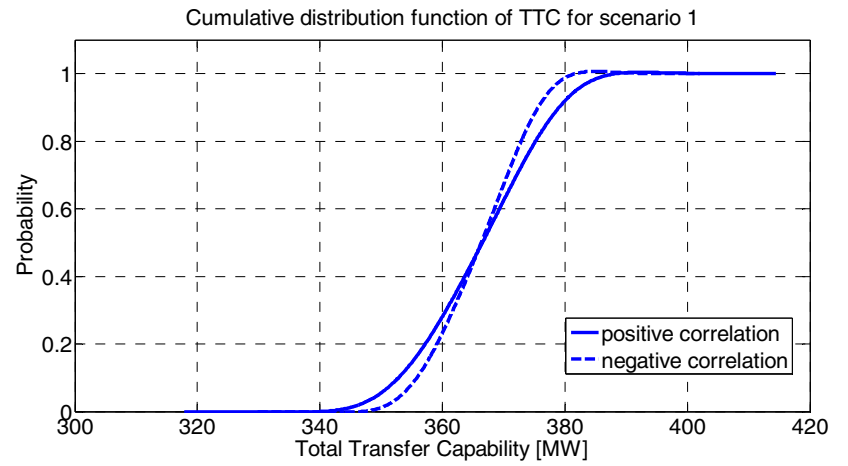

Fig. 3. CDF's of TTC for scenarios 1 (positive correlation) and 2 (negative correlation) for stress A

The level of correlation among RES plants also depends on the distance among the RES plants: the higher the distance, the lower the correlation. To this purpose, Fig. 4 compares the CDFs of scenarios 1 and 3 which differ for the distance among RES plants in areas 4 and 6; thus, also for correlation coefficients among relevant power injections are different ( 0.7 and 0.06 for scenarios A1 and A3). It can be noticed that in case of $\alpha=90 \%$ the NTC value reduces from 354 to 353 MW if the RES have a higher correlation: in fact, the larger the correlation, the higher the dispersion of the powerflows along the corridor due to larger displacements of conventional generators. This demonstrates that the proposed tool is able to quantify the effect of correlation of forecast errors on the final NTC value. Similar considerations hold comparing scenario 5 and 7 related to stress $\mathrm{B}$ : a larger distance between RES plants at both side of the interconnection between areas $1+2$ and areas $3+4$ determines an increase of the NTC from $756 \mathrm{MW}$ to $757 \mathrm{MW}$.

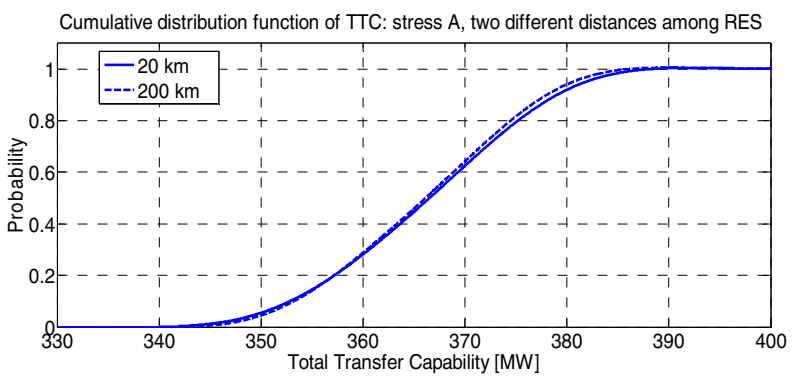

Fig. 4 Comparison of CDFs of TTC between Areas 4 (UP) and 6 (DOWN) for two distances among WFs: $20 \mathrm{~km}$ (dotted line) and $200 \mathrm{~km}$ (solid line)

Another influencing factor is the time horizon under analysis: in general, the larger the time horizon of analysis the less accurate is the forecast, thus the larger is the standard deviations of the forecast error. This statement can encounter some counterexamples: forecasts may sometimes be less accurate on shorter time horizons than on longer time horizons, due to specific conditions under which short term forecasts are performed, and to the forecast technique adopted.

For each RES unit (Wind turbine and Solar plant) the present methodology assumes a linear relationship between the curve provides the derating factor $\sigma_{k-\text { hours }} / \sigma_{24-\text { hours }}$ estimated from [9] which links the standard deviation $\sigma$ of forecast error for a WT to forecast time horizon.

Fig. 5 compares the CDFs of the TTC between areas $4+3$ and $1+2$ for scenarios B5 and B8 with time horizons equal to 12 and 24 hours. Assuming a value equal to $90 \%$ for parameter $\alpha$ it can be noticed that NTC value passes from 756 to 758 when time horizon passes from 24 to 12 hours.

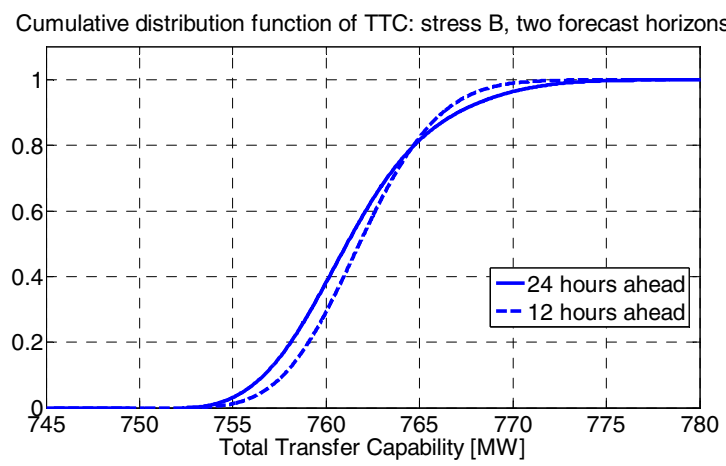

Fig. 5 Comparison of CDFs of TTC between areas $1+2$ (DOWN) and $3+4$ (UP) for two time horizons: 12 hours (dotted line) and 24 hours (solid line)

The same consideration holds valid for scenarios A: given $=90 \%$ for A1 (24 hours ahead forecasts) and A4 (12 hours ahead forecasts) the NTC values are respectively equal to 352 and 355 MW.

\section{Effect of period of the year and time of day}

This subsection intends to investigate the effect of the different period of the year (and hour of the day) in determining the solar generation forecast error modeling. The 
same level of production of a solar plant in different periods of the year (e.g. spring and summer) determine different clearness indexes, because the maximum producible power differs between seasons. This in turn causes a different derating factor from Fig. 1, thus a different standard deviation of the forecast error. To demonstrate this, Fig. 6 compares the MW injections of Solar Plants in $2 m+1$ PEM states for scenarios 1 and 9 (stress A) which differ only for the month of the year (March in A9 and August in A1). It can be noticed that the maximum excursions of solar production on PV2 passes from $80 \mathrm{MW}$ for August case to about $20 \mathrm{MW}$ for March case: this determines a larger standard deviation for the TTC curve in "August" scenario A1, and NTC estimate passes from $352 \mathrm{MW}$ (in A1) to $354 \mathrm{MW}$ (in A9).

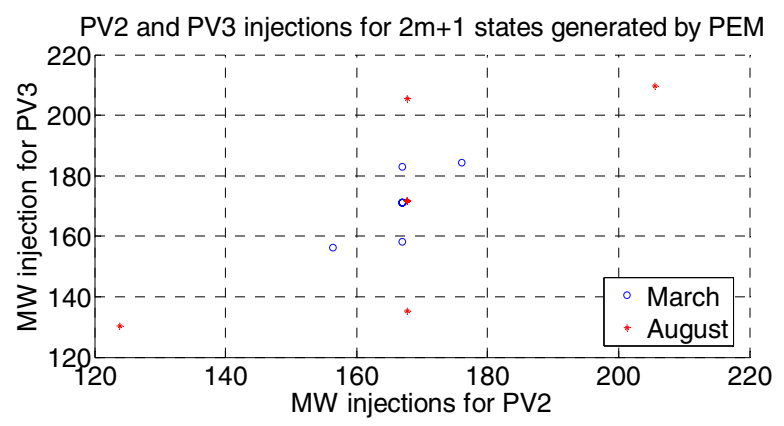

Fig. 6. Comparison of the MW solar injections in the $2 \mathrm{~m}+1$ PEM states: March (blue circles) and August (red *)

Similar consideration can be made for stress B, comparing simulation scenarios $\mathrm{B} 5$ and $\mathrm{B} 10$ : in scenario B10, one of solar injections PV2 has a clearness index equal to 1, i.e. the active power assignment to this RES plant is equal to the maximum producible power at $1 \mathrm{pm}$ in March. This implies that its standard deviation is drastically reduced. As a consequence, the CDF of the TTC undergoes a reduction of its standard deviation from scenario "August" scenario to "March scenario" and the NTC estimate passes from 756 MW in scenario B5 to $760 \mathrm{MW}$ in scenario B10 (see Fig. 7).

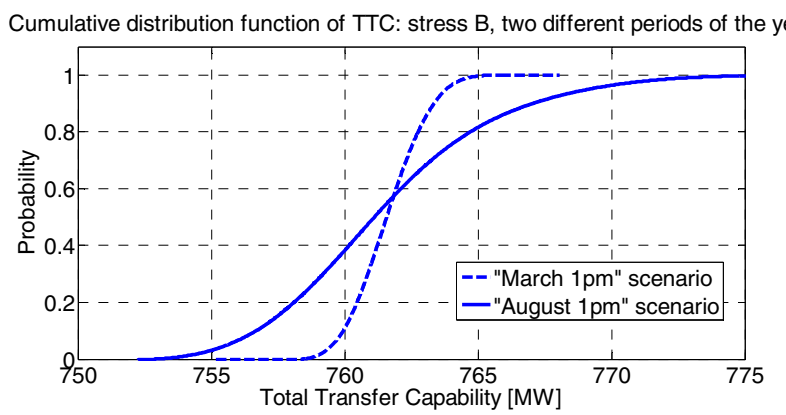

Fig. 7. Comparison of CDFs of TTC between areas $1+2(\mathrm{DOWN})$ and $3+4$ (UP) for two periods of the year: August at $1 \mathrm{pm}$ (solid line) and March at $1 \mathrm{pm}$ (dashed line)

\section{CONClusions}

The paper proposed an innovative application of the combination of PEM and TPN Transformation for probabilistic assessment of Net Transfer Capacity between contiguous areas in the power system. The advantage of the proposed method with respect to the conventional approach is that the reliability margin is not a fixed value (in MW or in \% of the TTC), but it is accurately determined as a function of the uncertainties due to RES forecast errors over the time horizon under study for operational planning purposes.

Even though simulations are carried out on a small test system with a low RES penetration case $(15 \%$ of load coverage by RES) and realistic values (around 10\%) for the standard deviations of the RES injection forecast errors, the results show that NTC is affected -to different extents- by various influencing factors: the most significant effects on NTC come from the sign of correlation coefficients among forecast errors and from the period of the year and of the day, which characterize solar generation forecast error models. In general terms, the extensive testing reaches the goal to prove the effectiveness of the proposed methodology which can catch the effect of many influencing factors, such as the time horizon, the distance among RES plants, the period of the year and the hour of day, and the correlation sign. The proposed method allows a more precise determination of the transfer capacity allowable for market transactions, avoiding under or over-estimations often brought by conventional NTC computations for capacity allocations on ATC based markets.

\section{APPENDIX - POINT EsTIMATE METHOD AND THIRD ORDER POLYNOMIAL NORMAL TRANSFORMATION}

\section{A. The $2 m+1$ PEM scheme}

Given $m$ stochastic variables $X$ as inputs of a scalar function $U$, the PEM Scheme generates $2 m+1$ points, which are used to compute the values of $U$ and are given by:

- two vectors for each stochastic variable:

$$
\bar{X}_{l, k}=\left(\mu_{1}, \mu_{2}, \ldots, \bar{x}_{l, k}, \ldots, \mu_{m}\right) \quad k=1,2
$$

where $l$ identifies the stochastic variable, $\mu_{i}(i=1, \ldots, m, \quad l \neq i)$ is the mean of $i$-th input, $\bar{x}_{l, k}$ is defined as: $\bar{x}_{l, k}=\mu_{l}+\xi_{l, k} \cdot \sigma_{l}$ where $\sigma_{l}$ is the standard deviation of $l$-th variable, and $\xi_{l, k}$ is given by:

$$
\xi_{l, k}=\frac{\lambda_{l, 3}}{2}+(-1)^{3-k} \cdot \sqrt{\lambda_{l, 4}-\frac{3 \lambda_{l, 3}^{2}}{4}}, \quad k=1,2 ; \xi_{l, 3}=0
$$

with $\lambda_{l, 3}$ and $\lambda_{l, 4}$ representing the normalized third and fourth statistical moments, i.e. $\lambda_{l, 3}=\sigma_{l, 3} / \sigma_{l}^{3}$ and $\lambda_{l, 4}=\sigma_{l, 4} / \sigma_{l}^{4}$ where $\sigma_{l, 3}, \sigma_{l, 4}$ are respectively the central moments of $3^{\text {rd }}$ and $4^{\text {th }}$ order for $l$-th variable

- one vector of mean values $\mu_{i}(i=1, \ldots, m)$ for all stochastic variables

The moments necessary to define the CDF of the resulting function $U$ are calculated as: $E\left(U^{j}\right)=\sum_{l=1}^{m} \sum_{k=1}^{3} w_{l, k} \cdot U(l, k)^{j}$ where weights $w_{l, k}$ are obtained by:

$$
w_{l, k}=\frac{(-1)^{3-k}}{\xi_{l, k} \cdot\left(\xi_{l, 1}-\xi_{l, 2}\right)}, \quad k=1,2 ; \quad w_{l, 3}=\frac{1}{m}-\frac{1}{\lambda_{l, 4}-\lambda_{l, 3}^{2}}
$$




\section{B. TPN Transformation}

This transformation permits to extend the potentialities of probabilistic power flow also to dependent non-normal distributions. Given marginal distributions $F_{X}$ of inputs $\boldsymbol{X}$, the basic idea is to express non normal variables $\boldsymbol{X}(i=1, \ldots, m)$ as a third order polynomial of dependent normal variables $Z$ :

$$
x_{i}=a_{0, i}+a_{1, i} \cdot Z_{i}+a_{2, i} \cdot Z_{i}^{2}+a_{3, i} \cdot Z_{i}^{3} \quad i=1, \ldots, m
$$

Coefficients $a_{0, i}, a_{1, i}, a_{2, i}, a_{3, i}$ are calculated as functions of

$L$-moments $\ell$ [24]. In particular:

$$
\left\{\begin{array}{c}
a_{0, i}=\ell_{1, i}-1.81379937 \cdot \ell_{3, i} \\
a_{1, i}=2.25518617 \cdot \lambda_{2, i}-3.93740250 \cdot \ell_{4, i} \\
a_{2, i}=1.81379937 \cdot \ell_{3, i} \\
a_{3, i}=-0.19309293 \cdot \ell_{2, i}+1.574961 \cdot \ell_{4, i}
\end{array}\right.
$$

$L$-moments are calculated as a function of the expectation value of an order statistic $E X[25]$ :

$$
\ell_{r}=\frac{1}{r} \sum_{k=0}^{r-1}(-1)^{k} \cdot\left(\begin{array}{c}
r-1 \\
k
\end{array}\right) \cdot E X_{r-k: r}
$$

where

$$
E X_{r-k: r}=\frac{r !}{(r-k-1) ! k !} \int_{-\infty}^{+\infty} x \cdot[F(x)]^{r-k-1} \cdot[1-F(x)]^{k} f(x) d x
$$

with $f$ and $F$ respectively the density function and the cumulative distribution function of stochastic variable $x$.

Also $\boldsymbol{Z}$ variables are dependent and the correlation matrix $\rho_{z}$ among them can be derived from $\rho_{X}$ by solving the equation below for any pair of $\boldsymbol{X}$ variables:

$6 a_{3, i} a_{3, j} \rho_{Z i, Z j}^{3}+2 a_{3, i} a_{3, j} \rho_{Z i, Z j}^{2}+\left(a_{1, i}+3 a_{3, i}\right)\left(a_{1, j}+3 a_{3, j}\right) \rho_{Z i, Z j}+$ $\left[\left(a_{0, i}+a_{2, i}\right)\left(a_{0, j}+a_{2, j}\right)-\rho_{X i X j} \sigma_{X i} \sigma_{X j}-\mu_{X i} \mu_{X j}\right]=0$

where $\sigma_{X i}, \mu_{X i}$ are respectively the standard deviation and the mean value of variable $i$. Again, $\rho_{Z}$ is positive definite and it can be subject to Cholesky decomposition. $G_{Z}$ is the resulting matrix so that $\rho_{Z}=G_{Z} G_{Z}^{T}$. Matrix $G_{Z}$ allows to transform dependent normal variables $\boldsymbol{Z}$ into independent normal variables $\boldsymbol{Y}$ to which the PEM can be applied. The transformation from $\boldsymbol{Z}$ to $\boldsymbol{Y}$ is: $Y=G_{Z}^{-1} \cdot Z$.

\section{REFERENCES}

[1] ENTSO-E (European Network of Transmission System Operators for Electricity), "R\&D Roadmap 2013-2022: Writing history again”, Dec. 2012

[2] D. Cirio, D. Lucarella, S. Massucco, "Online Dynamic Security Assessment to Mitigate the Risk of Blackout in the Italian Power System", European Transactions on Electrical Power (ETEP), Vol.18, No.8, Nov. 2008, pp. $784-801$

[3] H. Holttinen et al., "Design and operation of power systems with large amounts of wind power", IEA Wind Task 25, Final report, Phase I, 2006- 08, Helsinki, 2009

[4] S. Alessandrini, S. Sperati, P. Pinson, "The influence of new ECMWF Ensemble Prediction System resolution on wind power forecast accuracy and uncertainty estimation", Adv. Sci. Res., 8, 2012, pp. 143-147

[5] E. Ciapessoni, D. Cirio, A. Pitto, S. Massucco, F. Silvestro, "A Novel Approach to Account for Uncertainty and Correlations in Probabilistic
Power Flow", IEEE PES Innovative Smart Grid Technologies (ISGT), Istanbul, 12-15 Oct. 2014

[6] G. Salic, Y. Robours, "Impact of German Wind Generation Forecasts on Net Transfer Capacities", 8th International Conference on the European Energy Market (EEM), Zagreb, 25-27 May 2011

[7] ENTSO-E, "Net Transfer Capacities (NTC) and Available Transfer Capacities (ATC) in the Internal Market of Electricity in Europe", 2000

[8] J.W. Stahlhut and G.T. Heydt, "StochasticAlgebraic Calculation of Available Transfer Capability", IEEE Transactions on Power Systems, vol.22, pp 616-623, 2007

[9] C. Yali, B. Zhaohong and W. Xifan, "Study on calculation of probabilistic available transfer capability", . International Conference on Power System Technology, PowerCon 2002, vol.4, pp 2052-2056, 2002

[10] Z. Ruiyang, L. Guoqing and C. Houhe, "Study of Probabilistic Available Transfer Capability by Improved Particle Swarm Optimization", International Conference on Power System Technology, PowerCon 2006, pp 1-6, 2006.

[11] A. B. Rodrigues and M. G. Da Silva, "Probabilistic Assessment of Available Transfer Capability Based on Monte Carlo Method with Sequential Simulation", IEEE Transactions on Power Systems, vol.22, pp 484-492, 2007

[12] G.C. Ejebe, J.G. Waight, M. Sanots-Nieto, W.F. Tinney, "Fast calculation of linear available transfer capability", IEEE Transactions on Power Systems, vol. 15, pp 1112-1116, 2000

[13] CWE (Central Western Europe) Steering Committee, "CWE Enhanced Flow-Based MC feasibility report”, Tech. Report, March 15, 2011.

[14] E. Ciapessoni, D. Cirio, A. Pitto, S. Massucco, F. Silvestro, S. Grillo, "An Integrated Platform for Power System Security Assessment Implementing Probabilistic and Deterministic Methodologies", IEEE Systems Journal, Vol 7, Issue 4, pp. 845 - 853, Dec 2013

[15] E. Rosenblueth, "Point estimates for probability moments", Proc. Nat. Acad. of Sci., Vol 72, Issue 10, pp. 3812-3814, 1975

[16] H. Yang, B. Zou, "The Point Estimate Method using third order polynomial normal transformation technique to solve probabilistic powerflow with correlated wind source and load", 2012 Asia-Pacific Power and Energy Engineering Conference (APPEEC), March 2012

[17] ENTSO-E, "Definition of Transfer Capacities in liberalized Electricity Markets", 2001

[18] U. Focken, M. Lange, K. M"onnich, H.-P. Waldl, H. G. Beyer, A. Luig "A statistical analysis of the reduction of the wind power prediction error by spatial smoothing effects", J. Wind Eng. Ind. Aerodyn., Vol. 90, Issue 3, pp. 231-246, 2002

[19] E. Lorenz, J. Hurka, G. Karampela, D. Heinemann, H. Georg Beyer, M. Schneider, "Qualified Forecast of Ensemble Power Production by Spatially Dispersed Grid connected PV Systems", IEA Task 36, 2007

[20] CAISO, "Integration of renewable resources - Technical appendixes for California ISO Renewable Integration Studies", Tech. Report, Oct 2010

[21] E. Ciapessoni, D. Cirio, S. Massucco, A. Pitto, "An Innovative Probabilistic Methodology for Net Transfer Capacity Evaluation", 2015 Powertech, Eindhoven, NL, June 28-July 2, 2015

[22] P. Zhang and S. T. Lee, "Probabilistic Load Flow Computation Using the Method of Combined Cumulants and Gram-Charlier Expansion", IEEE Transactions on Power Systems, Vol. 19, No. 1, February 2004

[23] C. Grigg et al. "The IEEE Reliability Test System-1996. A report prepared by the Reliability Test System Task Force of the Application of Probability Methods Subcommittee", IEEE Trans. on Power System, Vol., no.3, pp. 1010-1020, Aug. 1999

[24] P.L. Liu, D. Kiureghian, "A Multivariate distribution models with prescribed marginals and covariances", Probab. Eng. Mech., Vol. 1, Issue 2, pp. 105-112, 1986

[25] J.R.M. Hosking, "L-moments: analysis and estimation of distributions using linear combinations of order statistics", Journal of the Royal Statistical Society, Series B 52, pp. 105-124, 1990 